\title{
Relaxation of twist helicity in the cascade process of linked quantum vortices
}

\author{
Simone Zuccher* \\ Department of Computer Science, University of Verona, Ca' Vignal 2, Strada Le Grazie 15, 37134 Verona, Italy \\ Renzo L. Ricca ${ }^{\dagger}$ \\ Department of Mathematics \& Applications, U. Milano-Bicocca, Via Cozzi 55, 20125 Milano, Italy \\ and BDIC, Beijing University of Technology, 100 Pingleyuan, Beijing 100124, People's Republic of China
}

(Received 26 May 2016; published 18 May 2017)

\begin{abstract}
By numerically solving the three-dimensional Gross-Pitaevskii equation we analyze the cascade process associated with the evolution and decay of a pair of linked vortex rings. The system decays through a series of reconnections to produce finally three unlinked, unfolded, almost planar vortex loops. Total helicity, initially zero, remains unchanged throughout the process. The gradual transfer from writhe (due to initial linking) to twist helicity, followed by a continuous relaxation of twist across scales during the evolution is shown to be a generic mechanism that consistently takes place on each individual component.
\end{abstract}

DOI: 10.1103/PhysRevE.95.053109

\section{INTRODUCTION}

In his famous paper Moffatt [1] showed that helicity, $\mathcal{H}$, a conserved quantity of classical ideal fluid mechanics [2] given by the integral of the scalar product of the velocity $\mathbf{u}$ and vorticity $\boldsymbol{\omega}=\boldsymbol{\nabla} \times \mathbf{u}$, i.e., $\mathcal{H}=\int \mathbf{u} \cdot \boldsymbol{\omega} d^{3} \mathbf{x}$, admits topological interpretation in terms of Gauss linking number. This work was then extended to include helicity contributions from each individual component [3,4]. The importance of helicity in the study of turbulence was soon recognized [5], but it took time to appreciate the importance of its geometric and topological decomposition in the analysis of physical properties of complex systems (see Refs. [6,7] for reviews). For a collection of $n$ distinct vortex tubes, helicity can be written as a discrete sum of contributions, given by

$$
\mathcal{H}=\sum_{i \neq j} \Gamma_{i} \Gamma_{j} L k_{i j}+\sum_{i} \Gamma_{i}^{2}\left(W r_{i}+T w_{i}\right),
$$

where $L k_{i j}$ is the (Gauss) linking number of the vortex centerlines $C_{i}$ and $C_{j}(i \neq j, i, j=1, \ldots, n)$, and $\Gamma_{i}, W r_{i}$, and $T w_{i}$ are, respectively, circulation, writhe, and total twist of the $i$ th vortex. The sum $W r_{i}+T w_{i}$ is commonly referred to as the Călugăreanu-White invariant $S L_{i}$ of the $i$ th vortex. $L k_{i j}$ and $S L_{i}$ are topological invariants, whereas $W r_{i}$ and $T w_{i}$ are global geometric quantities (for rigorous definitions see Sec. III below). Helicity can be therefore decomposed in terms of external helicity [the first term in the right-hand side of Eq. (1)] and internal helicity [the second term in the right-hand side of Eq. (1)], and the latter decomposed further in terms of writhe and twist helicity $\left(\Gamma_{i}^{2} W r_{i}\right.$ and $\Gamma_{i}^{2} T w_{i}$, respectively). These individual contributions help to understand helicity and energy transfers across reconnections and scales in both classical and quantum systems.

In this paper, following up previous work done by the present authors [8] and similar investigations done by colleagues $[9,10]$, we analyze the cascade process of two quantum vortex rings $V_{1}$ and $V_{2}$ initially linked to form a

\footnotetext{
*simone.zuccher@univr.it

†renzo.ricca@unimib.it
}

positive Hopf link (see Fig. 1), whose evolution is governed by the three-dimensional (3D) Gross-Pitaevskii equation (GPE) [11,12], given by

$$
\frac{\partial \psi}{\partial t}=\frac{\mathrm{i}}{2} \nabla^{2} \psi+\frac{\mathrm{i}}{2}\left(1-|\psi|^{2}\right) \psi
$$

for the complex wave function $\psi$ with background unit density $\left(|\psi|^{2} \rightarrow 1\right.$ as $\left.|x| \rightarrow \infty\right)$. To avoid difficulties with the regularization of velocity and helicity [9] we shall take Eq. (1) as our working definition of helicity and, as will be clarified by the particular choice of the reference frame for twist (see again Sec. III below), we shall refer to this quantity as Seifert helicity [10]. By extracting information from computed data, we analyze at the highest possible numerical accuracy the geometric and topological quantities present in Eq. (1), we determine how writhe and twist change individually and globally throughout the process, and assess external and internal helicity changes across reconnections.

\section{GOVERNING EQUATIONS}

GPE provides a rather accurate model to study quantum vortex reconnection under conservation of the Hamiltonian $E=K+I$, given by the sum of kinetic energy $K=\frac{1}{2} \int \nabla \psi \cdot \nabla \psi^{*} d^{3} \mathbf{x}$ and interaction energy $I=\frac{1}{4} \int(1-$ $\left.|\psi|^{2}\right)^{2} d^{3} \mathbf{x}$ (where $\psi^{*}$ denotes the $\psi$ complex conjugate). As is well known, Eq. (2) can be decoupled into the standard continuity equation and momentum equation by using the Madelung transformation $\psi=\sqrt{\rho} \exp (\mathrm{i} \theta)$. After some straightforward algebra, by taking real and imaginary parts of Eq. (2), we obtain

$$
\begin{gathered}
\frac{\partial \rho}{\partial t}+\frac{\partial\left(\rho u_{k}\right)}{\partial x_{k}}=0 \\
\rho\left(\frac{\partial u_{k}}{\partial t}+u_{l} \frac{\partial u_{k}}{\partial u_{l}}\right)=-\frac{\partial p}{\partial x_{k}}+\frac{\partial \tau_{k l}}{\partial x_{l}},
\end{gathered}
$$

with fluid density $\rho=|\psi|^{2}$, velocity $\mathbf{u}=\nabla \theta$, pressure $p=$ $\rho^{2} / 4$ and (so-called) quantum stress $\tau_{k l}=\frac{1}{4} \rho \frac{\partial^{2} \ln \rho}{\partial x_{k} \partial x_{l}}(k, l=$ $1,2,3)$. The term $\tau_{k l}$, negligible with respect to the pressure term at length scales much larger than the healing length $\xi=1$ (of the order of the vortex core), at much smaller scales 

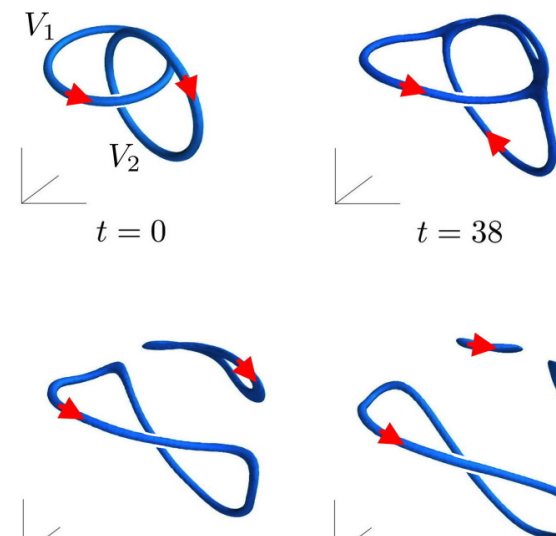

$t=47$

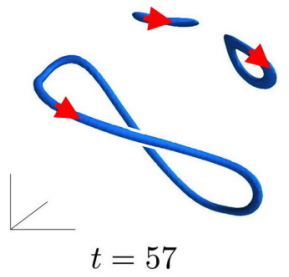

FIG. 1. Time sequence of the evolution of a system of vortex tubes governed by the Gross-Pitaevskii Eq. (2). Two initially linked vortex rings $V_{1}$ and $V_{2}$ move one against the other till a first reconnection takes place at time $t=36.5$. A single, folded vortex loop is generated and after a while it self-reconnects $(t=41)$ producing two loops. The smaller, secondary loop twists and self-reconnects further (at $t=50.5)$ to generate a third loop. No further reconnections are observed afterwards. The tubes are visualized by the isodensity surfaces given by $\rho=0.1$; the superimposed arrows (red online) indicate the vorticity direction.

becomes responsible of vortex reconnection [13] since it regularizes the momentum equation. At these microscopic scales reconnection can thus be regarded as a purely quantum effect.

\section{DEFINITION AND PHYSICAL INTERPRETATION OF GEOMETRIC AND TOPOLOGICAL PROPERTIES}

Quantum vortices are phase defects. As physical singularities of vorticity they can be regarded as zero density lines where phase is ill defined. However, as we shall see, physical effects associated with phase are all important and cannot be neglected by simply reducing vortex evolution to that of the vortex centerline. As in the classical case, the simultaneous presence of two or more vortices determine a superimposition of the induced velocity fields, that in the quantum case is reflected in the phase. In particular, the simultaneous presence of two noncoplanar, linked vortex rings (as in the case of our Hopf link) determines a mutually induced phase twist on each vortex, a signature of the complex topology of the ambient space. Note that this twist would not be present if the rings were not topologically linked.

To detect, visualize, and quantify phase information we rely on the fact that GPE vortices are fibered by isophase surfaces. We can then make use of these surfaces to define (unambiguously) a reference ribbon and, by applying a straightforward technique proposed in Ref. [8], to measure twist. The ribbon is defined by two edge curves: a baseline curve given by the vortex centerline $C$ and a second curve $C^{*}$ placed at a constant distance $\epsilon$ (the width of the ribbon) from $C$ along a unit vector $\hat{\mathbf{U}}$ orthogonal to the local unit tangent $\hat{\mathbf{T}}$ to $C$. The ribbon is then identified by the points of constant phase that lie on the isophase surface given by $\theta=\bar{\theta}$, this being given by the $\epsilon$ part of the surface bounded by $C$ and $C^{*}$.
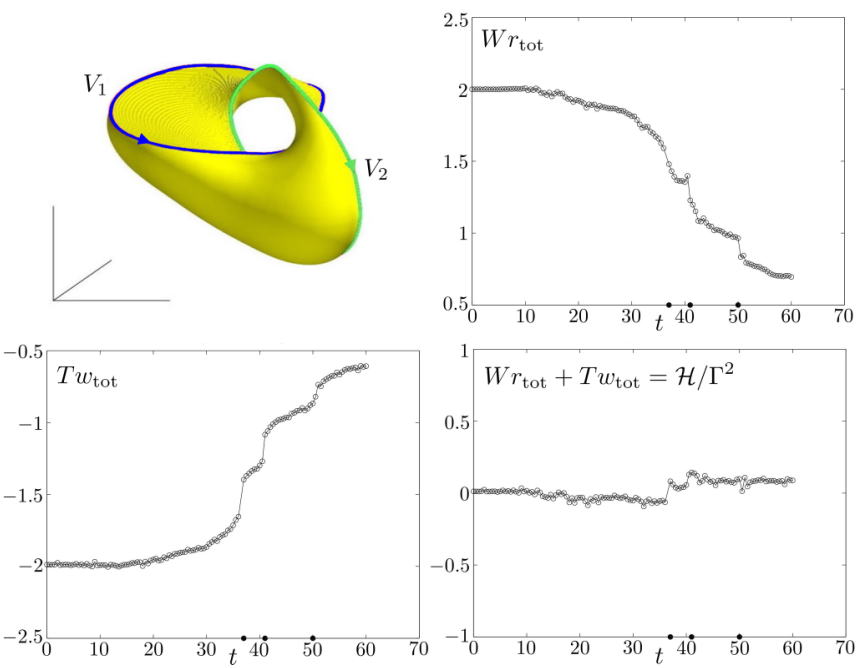

FIG. 2. A snapshot of the isophase surface $\theta=\pi$ (yellow) bounded by the vortex axes of $V_{1}$ (blue) and $V_{2}$ (green) at $t=23$ (i.e., before the first reconnection). Plots of total writhe (top right) $W r_{\text {tot }}$, sum of total twists (bottom left) $T w_{\text {tot }}$ and normalized total helicity [see Eq. (11)] during the cascade process of Fig. 1. Bullets on the time axis denote reconnection simulation times at $t=t_{1}=36.5, t_{2}=41$, and $t_{3}=50.5$.

By extending this isophase surface to the whole ambient space we have a (time-dependent) realization of a single fibration of the ambient space (see the example of Fig. 2, top-left plot, where the isophase surface $\theta=\pi$ at $t=23$ is shown).

For completeness we recall the standard definition of the geometric and topological quantities present in Eq. (1). The linking number between the vortices $i$ and $j$ is given by the double integral over the axes, given by

$$
L k_{i j}=\frac{1}{4 \pi} \int_{C_{i}} \int_{C_{j}} \frac{\mathbf{X}_{i}-\mathbf{X}_{j}}{\left\|\mathbf{X}_{i}-\mathbf{X}_{j}\right\|^{3}} \cdot\left(d \mathbf{X}_{i} \times d \mathbf{X}_{j}\right),
$$

where $\mathbf{X}_{i}$ and $\mathbf{X}_{j}$ denote the position vectors of a point on $C_{i}$ and $C_{j}(i \neq j)$, respectively. $L k_{i j}$ is a topological invariant of the link: it takes only integer values and in general it provides a measure of the degree of linking of two (or more) disjoint components. Hence it contributes to the external helicity of the disjoint union of two or more vortices.

The writhing number $W r_{i}$ is defined by

$$
W r_{i}=\frac{1}{4 \pi} \int_{C_{i}} \int_{C_{i}} \frac{\mathbf{X}_{i}-\mathbf{Y}_{i}}{\left\|\mathbf{X}_{i}-\mathbf{Y}_{i}\right\|^{3}} \cdot\left(d \mathbf{X}_{i} \times d \mathbf{Y}_{i}\right),
$$

where $\mathbf{X}_{i}$ and $\mathbf{Y}_{i}$ denote two distinct points on the same curve $C_{i} . W r_{i}$ is a global geometric property of $C_{i}$ and it takes real values. If $C_{i}$ is a plane curve, its writhe is always zero, but sometimes even fully twisted space curves may have writhe equal to zero (when positive and negative writhes compensate). Since writhe takes into account distortion, it is often a good indicator of three-dimensional folding.

Total twist is defined by the rate of rotation of the ribbon unit vector $\hat{\mathbf{U}}$ around the base curve $C_{i}$, i.e.,

$$
T w_{i}=\frac{1}{2 \pi} \int_{C_{i}}\left(\hat{\mathbf{U}} \times \frac{d \hat{\mathbf{U}}}{d s}\right) \cdot d \mathbf{X}_{i} .
$$


It is a global geometric property of the ribbon and it takes real values. $T w_{i}$ can be decomposed in terms of normalized total torsion $T_{i}$ and intrinsic twist $N_{i}$, given respectively by

$$
T_{i}=\frac{1}{2 \pi} \int_{C_{i}} \tau(s) d s,
$$

and

$$
N_{i}=\frac{1}{2 \pi} \int_{C_{i}} \frac{d \Theta(s)}{d s} d s=\frac{[\Theta]_{C_{i}}}{2 \pi},
$$

where $\tau=\tau(s)$ is local torsion (function of arc length $s$ ) of $C_{i}$, and $\Theta=\Theta(s)$ the angle between $\hat{\mathbf{U}}$ and the principal unit normal $\hat{\mathbf{N}}$ to $C_{i} . T_{i}$ takes real values, whereas $N_{i}$ is an integer measuring the total number of full turns of $\hat{\mathbf{U}}$ relative to the Frenet triad on $C_{i}$.

Since torsion computation is a source of great numerical noise [see, for example, Ref. [8] Fig. 5(b)] —especially across reconnections-we will not make use of this decomposition here. Rather, we shall compute twist by selecting and prescribing an isophase surface of our choice. Its computed value, being a closed integral over the whole fibration, is thus independent of the particular surface selected. The chosen surface is called a Seifert surface of the link, and it actually prescribes zero framing for the computation of linking numbers $[10,14,15]$, hence of helicity. Since the isophase surface is determined by the defect's topology, twist is a consequence of the topological complexity of the whole system. Twist, which for quantum vortices has no physical meaning, acquires physical justification through the mathematical realization of the twisted isophase surfaces associated with the wave function and therefore admits physical interpretation. Since by construction local twist is nonzero only when the gradient of the phase has a component directed along the vortex axis, and since $\mathbf{u}=\nabla \theta$, twist provides a measure of the longitudinal velocity field possibly present along the vortex axis. As in classical vortex dynamics, this contribution can indeed be quantified and written in terms of the tangential contribution to the velocity induced by the Biot-Savart law [10]. Without loss of generality we can take the ribbon to be on the isophase surface $\theta=\pi$ and unit width span (for visualization purposes we actually take $\epsilon=\xi / 2=0.5)$.

\section{NUMERICAL METHODS}

The numerical integration of the GPE (2) is carried out by employing a second-order Strang splitting Fourier spectral method. The computational domain is triply periodic. Since the initial condition is not periodic and we make use of the fast Fourier transform (FFT), boundary conditions are made periodic by doubling the computational domain in each direction with the introduction of mirror vortices. This well-known technique [16] increases the number of degrees of freedom eightfold. Since GPE conserves mass exactly, so does the method, a property often exploited in numerical simulations of vortex reconnection $[8,13,17]$.

\section{A. Initial conditions}

The initial condition is given by two initially circular, plane vortex rings and is prescribed as in Ref. [8]. Both
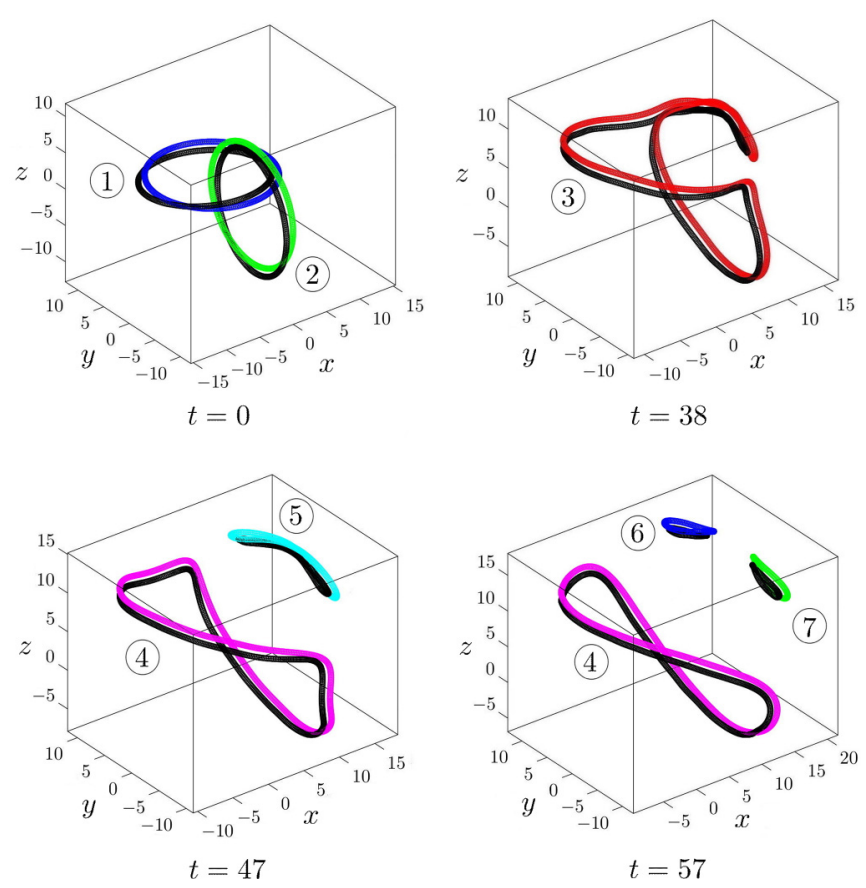

FIG. 3. Vortex center lines (in different colors), numbered sequentially after each reconnection event that marks a topological transition. The relative ribbon edge (in black) is always defined on the isosurface $\theta=\pi$.

vortices have equal circulation set to $\Gamma=2 \pi$. In order to avoid the time-consuming alternative approach based on prescribed induced velocity field, phase and density computation, and solution of the dissipative GPE, a new, higher-order Padé approximation given by $\rho_{0 k}(r)=\frac{a_{1} r^{2}+a_{2} r^{4}+a_{3} r^{6}+a_{4} r^{8}}{1+b_{1} r^{2}+b_{2} r^{4}+b_{3} r^{6}+a_{4} r^{8}}$ is used for the density distribution in the plane orthogonal to the vortex axis (see Ref. [18] for details), with an initial phase $\theta_{0 k}$ that changes by $2 \pi$ in that plane. By mirroring the wave function we ensure a periodic initial condition $\psi_{0}$ in both phase and density. The resulting wave function at $t=0$ is thus given by $\psi_{0}=\sqrt{\rho_{01} \rho_{02}} \exp \left[\mathrm{i}\left(\theta_{01}+\theta_{02}\right)\right]$. Initially the two rings have same radius $R_{0}=8$ and they are placed on mutually orthogonal planes, centered at $(0.5,4.5,0)$ in the $x-y$ plane and $(0,-4,0)$ in the $y-z$ plane (see Fig. $3, t=0)$. This asymmetric initial configuration is used to avoid possible simultaneous reconnections, giving a clear picture of the cascade process.

The computational domain is given by $[-20 ; 20] \times$ $[-25 ; 25] \times[-20 ; 30]$ and it is chosen to optimize the observed vortex evolution. The spatial discretization is the same in each direction, with resolution $\Delta x=\Delta y=\Delta z=$ $\xi / 3,150^{3}$ grid points in the physical domain (before mirroring), and time step $\Delta t=1 / 80=0.0125$.

\section{B. Postprocessing of numerical data}

Particular care has been put in the computation of the geometric information associated with the vortex centerline and ribbon. The overall spatial resolution is taken to be $\xi / 3$; a significantly greater resolution would compromise computational efficiency (mirroring increases eightfold the number of computational degrees of freedom). Since at a postprocessing stage the time-splitting Fourier spectral method 
for singular solutions of the GPE provides accurate evaluation of the solution between grid points [18], we evaluate $\psi$ at the much finer resolution of $\xi / 10$ only at points in the tubular region where $\rho \leqslant 0.2$ (for a vortex core diameter of, say, $\xi=1$, we take, for example, ten grid points). For this we have employed nonuniform fast Fourier transform (NFFT) (see Ref. [19]), a package originally written in C language and recently included in the interface INFFTM developed for MATLAB and GNU OCTAVE (see Ref. [20] for details).

Once $\psi$ is determined in the tube with sufficiently high spatial resolution we extract the axis, first by selecting the points whose density is very small, and then by weighting the points by taking $\bar{\omega}=|\omega| / \rho(|\omega| \neq 0$ on the vortex axis and identically zero elsewhere). Since $\bar{w}$ increases rapidly as one moves towards the tube axis, the weighted points collapse onto $C$; after reordering, by relying on the local high spatial resolution provided by INFFTM, a very smooth vortex centerline is generated. Arc-length derivatives are thus computed without any further regularization to obtain, for instance, the local Frenet triad $\{\hat{\mathbf{T}}, \hat{\mathbf{N}}, \hat{\mathbf{B}}\}$ given by the unit tangent, normal, and binormal vector. The ribbon edge $C^{*}$ is obtained from the tube axis $C$ (the ribbon baseline) in four steps: (i) a set of equispaced points is generated on the circle of radius $\epsilon=\xi / 2=0.5$, centered on $C$ and perpendicular to $\hat{\mathbf{T}}$; (ii) at each point, $\psi$ is evaluated by using INFFTM [20]; (iii) the phase is then interpolated at a constant value $\bar{\theta}=\pi$; (iv) the ribbon edge $C^{*}$, placed at $\epsilon=$ constant from $C$ on the isophase surface $\bar{\theta}=\pi$, is computed and width renormalized to 1 . By construction the ribbon is thus given by a smoothly varying function of arc length, that is then used to compute $T w$ by employing Eq. (7). Direct use of Eq. (7) by the ribbon technique, rather than by the decomposition $T w=T+N$, proves to be quite reliable and avoids the well-known difficulties represented by the computation of second- and third-order derivatives necessary for the estimate of torsion and the singular behavior at inflexion points [4].

\section{VORTEX CASCADE PROCESS: RESULTS}

The initially linked vortex rings $V_{1}$ and $V_{2}$ evolve and interact through a cascade process driven by a series of reconnections and consequent change of topology (see Fig. 1). After an initial, mutual distortion the rings collide and undergo a first reconnection at $t=t_{1}=36.5$, producing a single, large, folded loop that soon after it self-reconnects at $t_{2}=41$. As a result, two unlinked smaller loops are formed, propagating away while bending and twisting till one of them self-reconnects at $t_{3}=50.5$ producing even smaller vortex rings, which move quickly away from the region. No other reconnections are observed thereafter. We should remark that this observed cascade has generic topological features, but the precise geometry of the evolution is not at all generic, nor is it a direct consequence of the initial linking. In this respect the overall time evolution is strongly influenced by the initial conditions. This is quite evident from considerations on large-scale dynamics governed by the Biot-Savart induction law, where small changes in the initial configuration, for example due to curvature, are known to determine dramatic changes in geometric dynamics at later times. This explains why similar cascade processes have not been observed in other simulations with different initial conditions $[9,10]$.

We compute individual and total contributions to writhe, twist, and helicity. The initial total writhe $W r_{\text {tot }}=W r\left(C_{1} \# C_{2}\right)$ of the linked rings $C_{1} \# C_{2}$, before the first reconnection $(t<36.5)$, is given by the formula demonstrated in Ref. [21], that is,

$$
W r_{\text {tot }}=W r\left(C_{1} \# C_{2}\right)=W r_{1}+W r_{2}+2 L k_{12} .
$$

Since initially we have $W r_{1}=W r_{2}=0$ and $L k_{12}=L k_{21}=$ +1 , we have $\left.W r_{\text {tot }}\right|_{t=0}=+2$. The rings remain linked till the first reconnection takes place, and after that $L k_{12}=L k_{21}=0$ for all time. The time-dependent behavior of the total writhe $W r_{\text {tot }}$ is shown in the top-right diagram of Fig. 2. We notice an almost monotonic, progressive decrease of $W r_{\text {tot }}$ (allowing for numerical errors), with no appreciable jump in total writhe throughout the evolution. The small jiggles appearing around the reconnection times are presumably due to the presence of spurious numerical errors in the spatial neighborhood of the reconnection sites. However, careful refined local numerics indicate that writhe values just before and after each reconnection event appear to be unaffected by the topological transition, in agreement with the theoretical prediction of writhe conservation across reconnection based on analytical results [21]. However, noticeable changes in writhe rates seem to characterize the time frame of the reconnection events, where higher gradients are markedly more present. Since writhe is a geometric measure of three-dimensional coiling and folding, the decreasing tail evident in the plot is an indication that indeed the overall geometry of the system keeps changing, evolving towards less twisted structures. A similar trend can be observed also in the later stages of the decay of vortex links in water, measured by laboratory experiments [22].

The time-dependent behavior of the sum of total twists, given by $T w_{\text {tot }}=\sum_{i} T w_{i}$, is shown at the bottom of Fig. 2, together with the sum $W r_{\text {tot }}+T w_{\text {tot }}$, which by definition represent the normalized total helicity

$$
\frac{\mathcal{H}}{4 \pi^{2}}=W r_{\text {tot }}+T w_{\text {tot }}
$$

Similarly to total writhe, also $T w_{\text {tot }}$ is seen to decrease in absolute value monotonically. This seems partially consistent with what has been found for the helicity evolution of a pair of linked rings (see Ref. [9], Fig. 2, where $H=-\Gamma^{2} T w_{\text {tot }}$ ) in the time frame of the reconnection phase. On a longer time scale the different behavior might be due to the comparatively different core-to-size aspect ratio in $\xi$ units (see also Ref. [10], Fig. 5 and discussion below). As mentioned earlier, the mutual induction of vorticity at $t=0$ implies the presence of a longitudinal flow on each vortex ring; this is manifested by the presence of twist on each vortex, that compensates the external initial linking in the system. As a result the initial total helicity is equal to zero. From the bottom-right plot of Fig. 2, we see that $\mathcal{H} / 4 \pi^{2} \approx 0$ and it remains almost zero throughout the process. Hence, writhe conservation across reconnections implies total twist conservation across reconnections, as shown by direct inspection of the bottom-right plot of Fig. 2 (a result confirmed by the algebraic sum of the numerical values of the individual plots across each reconnection event, as shown in Fig. 4 below). In the GPE context it is the 

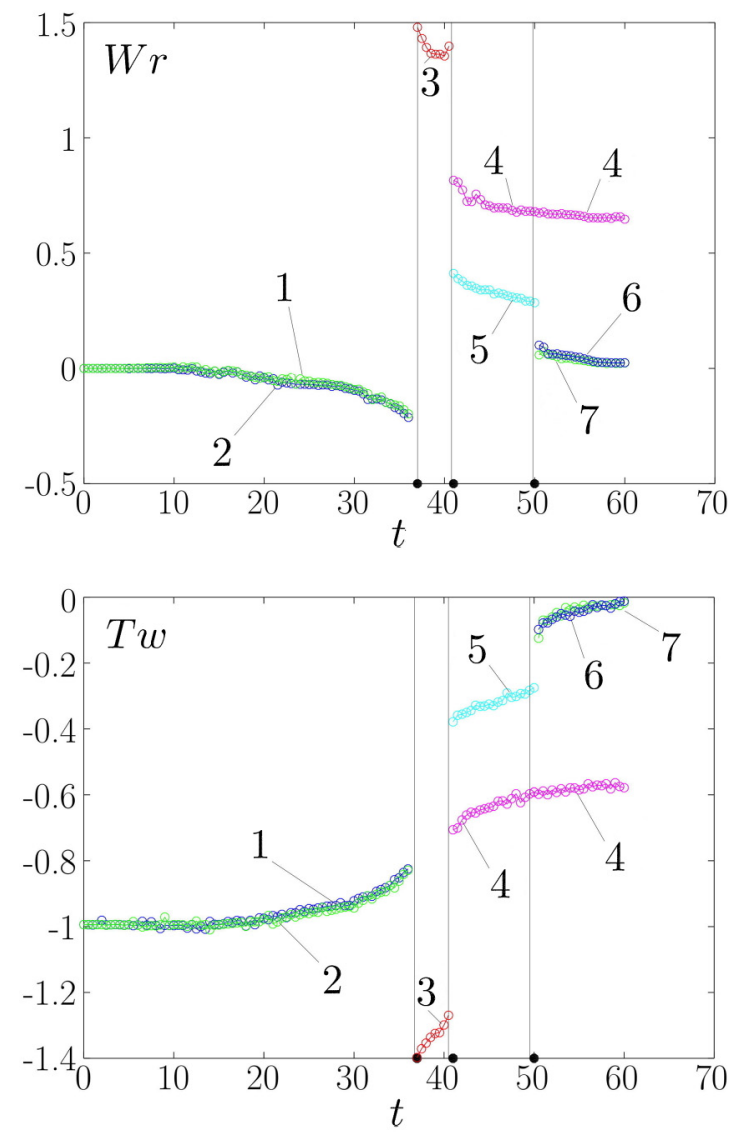

FIG. 4. Plots of individual writhe $W r$ and twist $T w$. Numbers refer to individual components at each stage of the cascade process. Bullets on the time axis denote the reconnection simulation times at $t=t_{1}=36.5, t_{2}=41, t_{3}=50.5$; vertical lines locate topological transitions.

Hamiltonian to remain conserved under reconnections [8], with conversion of kinetic energy to sound emission and Kelvin waves $[9,13,23,24]$. The fact that helicity appears to remain constant in spite of reconnections (the small fluctuations of order \pm 0.05 are presumably due to numerical errors) may be physically justified by the presumed negligible depletion of density core given by the small vortex core-to-size aspect ratio in relation to the box size (in $\xi$ units), in partial agreement with the observations made for the trefoil knot GPE simulation by Scheeler et al. (see Ref. [22], Fig. 5).

Visualization of the ribbon of each vortex component during evolution is shown in Fig. 3, where each component (denoted by different colors) is numbered sequentially after each topological transition (through reconnection). Individual ribbons are visualized by the individual vortex axes (with colored baselines) and ribbon edges in black. As mentioned in Sec. IV B the ribbons deform smoothly between reconnections allowing for an accurate computation of the individual contributions of writhe and twist, relative to each numbered component. As we see from the two plots of Fig. 4, individual writhe contributions consistently decrease between reconnections (allowing for numerical errors), accompanied by an equal and opposite increase of individual negative twist values. Hence, in absolute value individual twist keeps decreasing. Before the first reconnection (i.e., for $t<36.5) S L_{1}$ and $S L_{2}$ remain basically unchanged and equal to zero (not shown). For $t>$ 36.5 the two loops unlink $\left(L k_{i j}=0\right)$ and total linking remains essentially zero thereafter (with noticeable minor fluctuations due to numerical noise). The sudden jump at $t_{1}=36.5$ is signature of the unlinking process. After this the persistent decrease in writhe between reconnections corresponds to the relaxation of folded structures to almost planar vortex rings of smaller and smaller size, with gradual relaxation of total twist (in absolute value) of the newly formed structures. This appears to be a generic process that characterizes the whole cascade.

\section{CONCLUDING REMARKS}

A numerical simulation of the cascade process of a pair of initially linked quantum vortex rings governed by the Gross-Pitaevskii equation has been performed. Initially the vortex rings are identical and are placed in mutually orthogonal planes. Each vortex ring moves along its own central axis inducing a longitudinal flow on the other; hence both rings translate along, and rotate about their own axes with constant speed, with translation speed about twice that of rotation. After a first reconnection a long, single, folded loop is generated. A secondary reconnection leads to the formation of two disjoint, unlinked, twisted loops and the cascade process continues till one of the loops reconnects again to form a third small ring. No other reconnections are observed afterwards. At each stage we measure individual and total contributions to writhe, twist, and total helicity (here denoted Seifert helicity) by following the evolution of the isophase surface given by $\theta=\pi$, which provides a smoother computation of twist and other quantities. By this approach we avoid the computation of total helicity given by the integral formula and also the computation of total torsion for total twist, which otherwise would produce too much numerical noise (preventing also direct comparison with computation of the integral form of helicity). In this respect computation of Seifert helicity based on Eq. (1) provides a much nicer setting. At $t=0$ total helicity is zero in spite of the initial linking, and this because of the presence of a nonzero total twist in each ring; total helicity remains then essentially zero throughout the process (with some small fluctuations presumably due to numerical errors). Since GPE vortices are fibered by their isophase surfaces this result seems to be in good agreement with an earlier theoretical proposition on fibered knots [14]. As the system unlinks through the first reconnection we have a drop of external helicity compensated by an equal and opposite increase of internal helicity. During this unlinking phase total writhe drops and after the first reconnection, individual writhe keeps decreasing as a signature of the persistent, gradual evolution towards more planar structures. This is accompanied by a continuous relaxation of internal twist (in absolute value); hence, individual twist relaxation is found to be a generic mechanism regardless of the initial writhe value. Since total twist is due to contributions from total torsion and intrinsic twist, the progressive transition from linked to folded to unlinked, unknotted planar loops implies progressive decrease of total torsion, and depletion of kinetic energy into sound emission. Moreover, in agreement with theoretical predictions [21] total writhe is found to 
remain conserved across reconnections; since total helicity remains constantly zero, total twist remains also conserved across reconnections. This too seems to be in agreement with expectations, which would ascribe a change in helicity across reconnection to twist transfers [21]. In conclusion, the cascade process analyzed here is characterized by a generic production of almost planar, smaller vortex rings and by the redistribution and relaxation of total twist across scales. Since writhe is a good indicator of three-dimensional folding and total twist of torsional energy, our results indicate a preferential path towards the relaxation of folding to torsional energy across scales. Testing this preliminary conclusion against yet more elaborate cascade processes is a subject for future research.
[1] H. K. Moffatt, The degree of knottedness of tangled vortex lines, J. Fluid Mech. 35, 117 (1969).

[2] J.-J. Moreau, Constantes d'un îlot tourbillonnaire en fluid parfait barotrope, C.R. hebd Sèanc. Acad. Sci., Paris 252, 2810 (1961).

[3] R. L. Ricca and H. K. Moffatt, The helicity of a knotted vortex filament, in Topological Aspects of the Dynamics of Fluids and Plasmas, edited by H. K. Moffatt et al. (Kluwer, Dordrecht, 1992), pp. 225-236.

[4] H. K. Moffatt and R. L. Ricca, Helicity and the Călugăreanu invariant, Proc. R. Soc. London A 439, 411 (1992).

[5] R. Betchov, Semi-isotropic turbulence and helicoidal flows, Phys. Fluids 4, 925 (1961).

[6] R. L. Ricca and M. A. Berger, Topological ideas and fluid mechanics, Phys. Today 49(12), 28 (1996).

[7] H. K. Moffatt, Helicity and singular structures in fluid dynamics, Proc. Natl. Acad. Sci. U.S.A. 111, 3663 (2014).

[8] S.Zuccher and R. L. Ricca, Helicity conservation under quantum reconnection of vortex rings, Phys. Rev. E 92, 061001 (2015).

[9] P. Clark di Leoni, P. D. Mininni, and M. E. Brachet, Helicity, topology, and Kelvin waves in reconnecting quantum knots, Phys. Rev. A 94, 043605 (2016).

[10] R. Hänninen, N. Hietala, and H. Salman, Helicity within the vortex filament model, Sci. Rep. 6, 37571 (2016).

[11] L. P. Pitaevskii, Vortex lines in an imperfect Bose gas, Sov. Phys. JETP 13, 451 (1961) [J. Exptl. Theoret. Phys. (U.S.S.R.) 40, 646 (1961)].

[12] E. P. Gross, Hydrodynamics of a superfluid condensate, J. Math. Phys. 4, 195 (1963).

[13] S. Zuccher, M. Caliari, A. W. Baggaley, and C. F. Barenghi, Quantum vortex reconnections, Phys. Fluids 24, 125108 (2012).
[14] W. L. De Sumners, in Graph Theory and Topology in Chemistry, edited R. B. King and D. Rouvray (Elsevier, Amsterdam, 1987), pp. 3-22; also W. L. De Sumners, and R. L. Ricca (unpublished).

[15] R. Dennis and J. Hannay, Geometry of Călugăreanus theorem, Proc. R. Soc. London A 461, 3245 (2005).

[16] J. Koplik and H. Levine, Vortex reconnection in superfluid helium, Phys. Rev. Lett. 71, 1375 (1993).

[17] A. J. Allen, S. Zuccher, M. Caliari, N. P. Proukakis, N. G. Parker, and C. F. Barenghi, Vortex reconnections in atomic condensates at finite temperature, Phys. Rev. A 90, 013601 (2014).

[18] M. Caliari and S. Zuccher, arXiv:1603.05022 [math.NA] (unpublished).

[19] J. Keiner, S. Kunis, and D. Potts, Using NFFT 3 - A software library for various Non-equispaced Fast Fourier Transforms, ACM Trans. Math. Software 36, 19 (2009).

[20] M. Caliari and S. Zuccher, INFFTM: Fast evaluation of 3d Fourier series in MATLAB with an application to quantum vortex reconnections, Comput. Phys. Commun. 213, 197 (2017).

[21] C. E. Laing, R. L. Ricca, and W. L. De Sumners, Conservation of writhe helicity under anti-parallel reconnection, Sci. Rep. 5, 9224 (2015).

[22] M. W. Scheeler, D. Kleckner, D. Proment, G. L. Kindlmann, and W. T. M. Irvine, Helicity conservation by flow across scales in reconnecting vortex links and knots, PNAS 111, 15350 (2014).

[23] M. Leadbeater, T. Winiecki, D. C. Samuels, C. F. Barenghi, and C. S. Adams, Sound Emission Due to Superfluid Vortex Reconnections, Phys. Rev. Lett. 86, 1410 (2001).

[24] M. Leadbeater, D. C. Samuels, C. F. Barenghi, and C. S. Adams, Decay of superfluid turbulence via Kelvin-wave radiation, Phys. Rev. A 67, 015601 (2003). 